\title{
Effects of Effective Mass Discontinuity on the Conductance of Three-Dimensional Quantum Wires
}

\author{
Luis C. O. Dacal ${ }^{1}$ and Erasmo A. de Andrada e Silva ${ }^{2}$ \\ ${ }^{1}$ Instituto de Estudos Avançados - IEAv - CTA, C. P. 6044, 12228-001, São José dos Campos - SP, Brazil \\ ${ }^{2}$ Instituto Nacional de Pesquisas Espaciais - INPE, C. P. 515, 12201-970, São José dos Campos - SP, Brazil
}

\section{Received on 08 December, 2005}

\begin{abstract}
We calculate the conductance of three-dimensional semiconductor quantum wires considering different effective masses in the contacts and in the channel. We show that, with respect to the case with equal masses in the channel and in the contacts, the amplitude of the conductance oscillations increases if the electron effective mass in the channel is larger and decreases if it is smaller than in the contacts. Effects on the density of probability are also shown. These effects of the effective mass discontinuity are explained in terms of kinetic confinement and transmission coefficient modulation.
\end{abstract}

Keywords: Quantum wire; Ballistic transport; Effective mass discontinuity

\section{INTRODUCTION}

Some years ago, intense efforts were devoted to the understanding of the effective mass discontinuity effects on the electronic properties of semiconductor systems. A good example is the so called kinetic confinement. When a semiconductor layer is grown between two others of a different material with a zero conduction band offset, there is no potential well to confine the electrons in the central layer. On the other hand, a kinetic energy variation is brought about by the effective mass discontinuity and another kind of confinement can take place, namely, the kinetic confinement. It occurs when the heaviest effective mass is in the central layer, giving rise to a kinetic energy reduction related to the transverse free motion. This kind of phenomenon was firstly exploited by Sasaki [1] in a proposal for an effective mass superlattice. After that, Kubisa and Zawadzki [2] investigated the effects of external magnetic fields on a 'kinetic well'. Since then, it has been investigated from the theoretical $[3,4]$ and experimental $[5,6]$ points of view. More recently, this interest was renewed through proposals for spintronic devices that employ different materials for contacts and channel $[7,8]$.

In this work, effects of the effective mass discontinuity are considered in a quantum wire system. We calculated the conductance for different ratios between the electronic mass in the contacts and in the channel. It is shown that this discontinuity determines the conductance oscillation amplitude. It also affects the electronic density of probability inside the channel and we employed a simple one-dimensional model to discuss the transmission coefficient dependence on the mass ratio. The conductance calculation is analogous to that performed in a previous work [9] where we analyzed the effects of the system dimensionality.

\section{MODEL}

We consider a three-dimensional hard wall channel, or quantum wire, that connects the source and drain regions (see the inset in Fig. 1). The mode matching technique is employed to calculate the quantum ballistic conductance in the effective mass and envelope function frameworks. We use a basis set

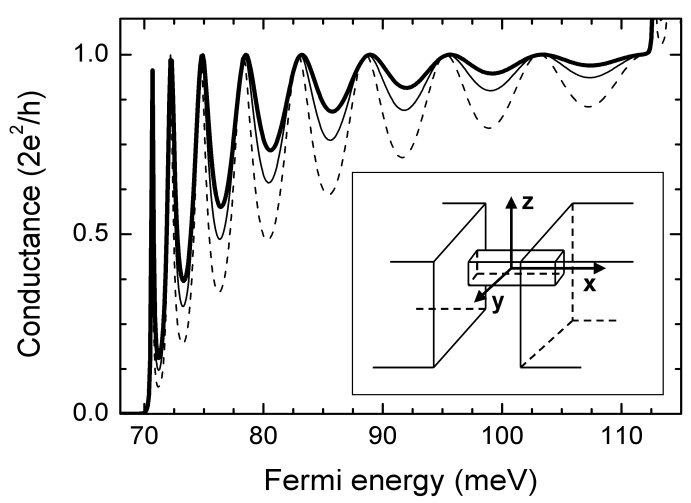

FIG. 1: Conductance as a function of Fermi energy for a $100 \mathrm{~nm}$ long quantum wire with a $10 \times 20 \mathrm{~nm}^{2}$ transverse section area. Three values for $r_{m}\left(m_{c} / m_{w}\right)$ are considered: 0.8 (dashed line), 1.0 (full line) and 1.2 (thick line).Inset: schematic representation of the system. The quantum wire is the central structure that connects the contacts. The axes orientation is also indicated.

with infinite quantum well solutions for the confined dimensions and plane waves for the propagating states. For more details, see our previous work [9] where the wave functions are presented and the calculation method is detailed.

Here, we take into account the difference between electronic effective masses in the quantum wire $\left(m_{w}\right)$ and in the contacts $\left(m_{c}\right)$, whose ratio is given by $r_{m}\left(r_{m}=m_{c} / m_{w}\right)$. In this work, we fix $m_{w}=0.067(\mathrm{GaAs})$ and change $m_{c}$ through $r_{m}$.

We would like to stress that in our system there is no kinetic energy reduction related to the transverse free motion. On the contrary, in a quantum wire, the transverse dynamics is quantized and the effective mass variation mainly affects the longitudinal motion through the probability flux continuity and energy conservation. 


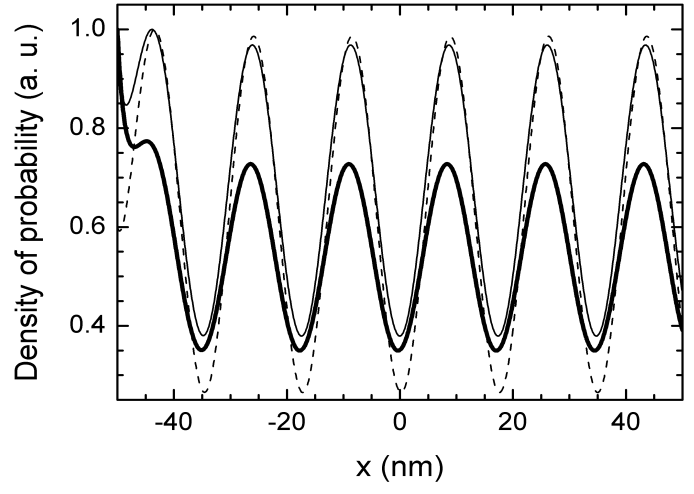

FIG. 2: Density of probability inside the quantum wire for the system considered in Fig. 1. The same $r_{m}$ values and correspondent lines are used. The Fermi energy is $88.7 \mathrm{meV}$.

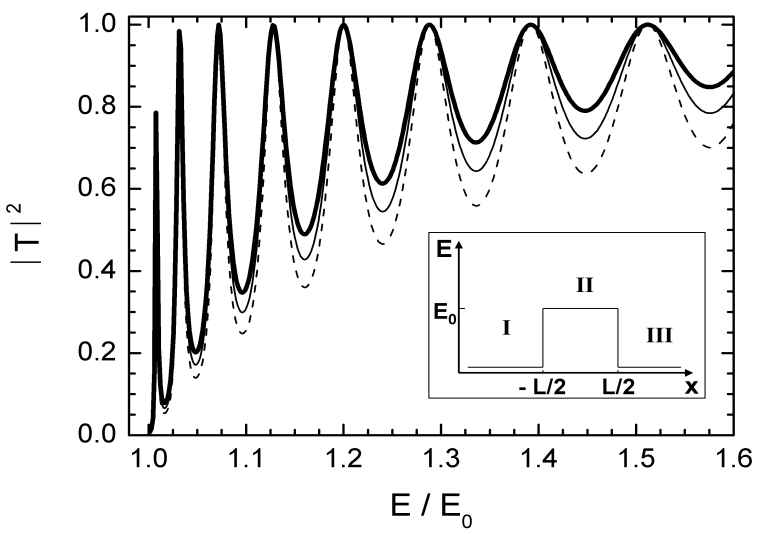

FIG. 3: Transmission coefficient as a function of Fermi energy for the one-dimensional analogue of the system considered on Fig. 1. The same $r_{m}$ values and correspondent lines are used. Inset: schematic representation of the one-dimensional system. $L$ corresponds to the quantum wire length and $E_{0}$ corresponds to the conductance threshold energy.

\section{RESULTS AND DISCUSSION}

In Fig. 1, we show the conductance as a function of the electronic Fermi energy for a $100 \mathrm{~nm}$ long quantum wire with a $10 \times 20 \mathrm{~nm}^{2}$ transverse section area. We consider three values for $r_{m}$, namely, 0.8 (dashed line), 1.0 (full line) and 1.2 (thick line). As one can see, the conductance oscillation amplitude goes with the inverse of $r_{m}$. In other words, one can say that the conductance oscillations are reinforced when $m_{w}>m_{c}$. This effect is analogous to the one observed in our previous work [9] where narrow quantum wires and their correspondent strong confinement also increased the conductance oscillation amplitude. The difference is that, here, the electronic confinement is not controlled by the wire width, but by the effective mass difference. Following this analogy, we may conclude that the effective mass discontinuity represents an extra electron scattering mechanism at the entrance and exit of the quantum wire.

At the same time, it is important to notice that the conductance oscillations and plateaus are present even when $r_{m}$ is greater than 1. This means that the qualitative aspects of the quantum wire conductance are preserved in this case.

In order to clarify the effects of effective mass variation on the electronic properties of the quantum wire, in Fig. 2, we show the density of probability as a function of the longitudinal quantum wire coordinate for the previous wire dimensions and three $r_{m}$ values. We used the same legend of Fig. 1 and $88.7 \mathrm{meV}$ for the Fermi energy, which corresponds to a conductance peak. It is shown that the charge localization is more pronounced for the least $r_{m}$ value, namely 0.8 (dashed line). This can be understood as a kind of charge confinement due to mass discontinuity when $m_{w}$ is greater than $m_{c}$. It is important to notice that the conductance threshold is not affected by $r_{m}$ due to the constant $m_{w}$ value. Moreover, the oscillatory behavior of the density of probability is weakened but preserved when $r_{m}$ is greater than 1 .

The quantum wire can be thought as a Fabry-Pérot like oscillator [9], what give us an alternative approach to explain the $r_{m}$ influence on the wire conductance. By using a simple one-dimensional model, an analytical expression for the transmission coefficient with explicit dependence on the $r_{m}$ factor can be obtained which shows the origins of the oscillation amplitude variation. This model is schematically represented on the inset of Fig. 3, where $L$ corresponds to the quantum wire length. The system is divided into three regions. I and III represent the contacts and II corresponds to the wire itself. In this last region, there is an energy barrier, $E_{0}$, that represents the conductance threshold. We considered plane waves in the three regions and imposed wave function and probability flux continuity at the interfaces. The energy conservation was also considered and the expression for the transmission probability is written as:

$$
|T|^{2}=\frac{\left(2 \cdot r_{m} \cdot K_{I} \cdot K_{I I}\right)^{2}}{\sin ^{2}\left(K_{I I} \cdot L\right) \cdot\left(r_{m}^{2} \cdot K_{I I}^{2}+K_{I}^{2}\right)^{2}+\cos ^{2}\left(K_{I I} \cdot L\right) \cdot\left(2 \cdot r_{m} \cdot K_{I} \cdot K_{I I}\right)^{2}}
$$

where $K_{n}$ represents the linear momentum in region $n$.
In Fig. 3, we show this transmission coefficient as a func- 
tion of the Fermi energy for a system equivalent to that one considered on Fig. 1, what means $L=100 \mathrm{~nm}$ and $E_{0}=70.15$ $\mathrm{meV}$. As expected, it reproduces the conductance dependence on $r_{m}$. This approach allows us to say that the effective mass variation has a significant effect on the transmission and reflection coefficients of the system. $r_{m}$ values lower than 1 $\left(m_{w}>m_{c}\right)$ reinforce these coefficients oscillations.

\section{CONCLUSIONS}

We showed that the conductance of semiconductor quantum wires is strongly affected by the effective mass discontinuity when the contacts and the channel are made of different materials. We employed the mode matching technique in the effective mass and envelope function frameworks to calculate the conductance in the ballistic regime. Our results show that the conductance oscillations amplitude is increased when the electronic mass in the wire is greater than that in the contacts. The same behavior is observed for the density of probability and the transmission coefficient, which was obtained through a simplified one-dimensional model.

We hope that our results may help to understand future experimental data on transport properties of systems composed by different materials.

\section{Acknowledgments}

This work was supported by the Brazilian agencies FAPESP, CNPq and FUNCATE.
[1] Akio Sasaki, Phys. Rev. B 30, 7016 (1984).

[2] M. Kubisa and W. Zawadzki, Semicond. Sci. Technol. 8, S246 (1993).

[3] D. Indjin, V. Milanović, and Z. Ikonić , Phys. Rev. B 52, 16762 (1995).

[4] J. Kim, L. W. Wang, and A. Zunger, Phys. Rev. B 56, R15541 (1997).

[5] D. Washington, T. Hogan, P. Chow, T. Golding, C. Littler, and U. Kirschbaum, J. Vac. Sci. Technol. B 16, 1385 (1998).
[6] D. W. Stokes, T. Hogan, P. Chow, T. Golding, U. Kirschbaum, C. Littler, and R. Lukic, J. Crystal Growth 201/202, 854 (1999).

[7] S. Datta and B. Das, Appl. Phys. Lett. 56, 665 (1990).

[8] I. Žutić, J. Fabian, and S. Das Sarma, Rev. of Modern Phys. 76 323 (2004)

[9] Luis C. O. Dacal, Álvaro J. Damião, and Erasmo A. A. Silva, Phys. Rev. B 71, 155330 (2005). 